Ildeberto Muniz de Almeida

(iD) https://orcid.org/0000-0002-8475-3805

a Universidade Estadual Paulista Júlio de Mesquita Filho (Unesp), Faculdade de Medicina de Botucatu. Botucatu, SP, Brasil.

Contato:

Ildeberto Muniz de Almeida

E-mail:

ildeberto.almeida@unesp.br

O autor informa que este trabalho não é baseado em tese ou dissertação e que não foi apresentado em evento científico.

O autor declara que o estudo não foi subsidiado e que não há conflitos de interesses.

\section{Proteção da saúde dos trabalhadores da saúde em tempos de COVID-19 e respostas à pandemia}

\author{
Health protection for healthcare workers in \\ COVID-19 times and responses to the pandemic
}

\section{Resumo}

Objetivo: discutir desafios para a elaboração e implementação de planos de resposta e prontidão estratégica contra a COVID-19. Métodos: partindo de recomendações da Organização Mundial da Saúde, que enfatiza três grupos de objetivos a serem buscados nesses planos, defende-se a inclusão do tema da proteção à saúde dos trabalhadores da Saúde no âmbito de seu terceiro objetivo: "Minimizar o impacto da epidemia sobre sistemas de saúde". Discussão: a ênfase dada ao uso de EPI, medidas de etiqueta social e higiene é discutida como potencialmente minimizadora da importância de medidas de controle de engenharia e de controles administrativos para a prevenção da doença. Destaque é dado à necessidade de treinamentos que formem trabalhadores para o reconhecimento de situações de risco associadas às dificuldades das novas atividades surgidas na pandemia, com ênfase em novas interações entre colegas e entre trabalhadores, assim como em novos instrumentos e contextos dela decorrentes. Os custos a serem pagos na forma de impactos na saúde dos trabalhadores tendem a crescer em contextos de degradação de situações de trabalho associados à rápida evolução da doença. Um dos desafios dos programas de resposta é o de reconhecer e responder com rapidez às mudanças que criam novos riscos.

Palavras-chave: trabalhadores da saúde; COVID-19; doenças infecciosas; saúde do trabalhador.

\begin{abstract}
Objective: to discuss challenges for developing and implementing response and strategic readiness plans against COVID-19. Methods: based on the World Health Organization recommendations, which emphasizes three groups of objectives to be pursued, we advocate including the topic of health protection for health workers within the scope of its third objective: "Minimize the impact of the epidemic on health systems." Discussion: the central argument adopted is that the emphasis on the use of PPE, social etiquette and hygiene measures can potentially minimize the importance of the engineering and administrative controls measures in preventing the disease. We highlight the need of training workers to recognize risk situations associated with the difficulties of the new activities that emerged during the pandemic, with emphasis on new interactions between healthcare professionals and also between other workers, as well as on new instruments and contexts related to the epidemic situation. The costs of the impacts on workers' health tend to increase due to degradation of work conditions and the fast evolution of the disease. One of the response programs challenges is how to recognize and respond quickly to the changes that lead to new risks.
\end{abstract}

Keywords: health workers; COVID-19; infectious diseases; occupational health. 


\section{Introdução}

No dia 31 de dezembro de 2019 a Organização Mundial de Saúde (OMS) foi informada, na China, sobre casos de pneumonia de etiologia desconhecida na cidade de Wuhan, Província de Hubei. O agente causal da doença foi, posteriormente, denominado SARS-CoV-2. No final de janeiro de 2020 havia 7.734 casos confirmados na China e 90 registrados em outros países. A taxa de mortalidade calculada era de 2,2\%. A situação foi declarada emergência de Saúde Pública de Interesse internacional ${ }^{1,2}$.

A importância da doença é ilustrada na magnitude alcançada e na rapidez de sua disseminação. No dia 25 de maio de 2020, a Organização PanAmericana de Saúde anunciava 5.304.772 casos confirmados no mundo, com 342.029 mortes $^{3}$.

No Brasil, o primeiro caso foi confirmado no dia 26 de fevereiro e em 3 de março havia cerca de 500 casos suspeitos. A atualização do dia 25 de maio de 2020, do Painel Coronavírus, do Ministério da Saúde informava: casos e óbitos confirmados, números acumulados: 374.898 e 23.473 , respectivamente ${ }^{4}$.

O alcance dos registros oficiais e o reconhecimento de casos relacionados ao trabalho preocupam. No Brasil, nem toda Doença Infecciosa e Parasitária (DIP) é de notificação compulsória e integra o Sistema de Informação de Agravos de Notificação (Sinan). Entre as doze DIP notificáveis e incluídas na lista brasileira de doenças relacionadas ao trabalho apenas quatro contam com o campo ocupação na ficha de notificação no Sinan. As demais não permitem registro desse dado. Além disso, a notificação de casos internados com suspeita de COVID-19 integra o registro das síndromes gripais que incluem as síndromes respiratórias agudas graves nem sempre especificadas ${ }^{5}$.

Este texto explora preocupações com a situação acima citada com ênfase em aspectos da saúde de trabalhadores da saúde, sem perder de vista outras categorias potencialmente afetadas.

Em primeiro lugar será abordado o tema dos planos de resposta em serviços de saúde ${ }^{6-8}$ à pandemia explorando aspectos de seus conteúdos e das bases em que se assentam. Atenção é chamada para o fato de que a OMS, instituições do campo da Saúde Pública e a literatura tratam do tema e podem subsidiar debates de comitês de resposta à pandemia e incentivar o uso de lições aprendidas no enfrentamento de crises anteriores.

Em segundo lugar, aborda-se o tema dos princípios éticos nas decisões relativas a criação, composição e instalação desses comitês e nas escolhas realizadas no desenho dos respectivos planos. Até que ponto respeitam a compreensão de que sistemas de saúde são complexos, incluindo grande variedade de categorias profissionais e serviços cujos interesses se não contemplados podem ter implicações éticas e políticas e alimentar resistências à implantação dos planos aprovados ${ }^{9,10}$.

Um terceiro aspecto é o da inclusão do tema da proteção da saúde dos trabalhadores (ST) da saúde nos planos de resposta (PR) à pandemia. A proteção da ST deve estar especificada no PR. A equipe dos Serviços Especializados em Engenharia de Segurança e em Medicina do Trabalho (SESMT) deve integrar o comitê e ser parte ativa na construção desse plano, guiando-se pela noção de hierarquia de medidas de prevenção e apresentando o uso adequado de equipamentos de proteção individual (EPI), medidas de etiqueta social e higiene de mãos como partes do plano ${ }^{5,7,11}$.

As medidas de proteção precisam conter orientações sobre como agir em contexto de trabalho que evolui mudando em grande velocidade e criando situações novas de risco e incerteza a serem adequadamente manejadas.

A chegada da pandemia de COVID-19 ao país e a forma como vem evoluindo criam novos desafios a pesquisadores e profissionais envolvidos com o tema da saúde dos trabalhadores da saúde, mas não só. No momento em que este texto está sendo finalizado crescem indícios de maior proximidade de cenário de saturação da capacidade dos serviços de saúde. Para os profissionais da saúde isso tende a significar o pior dos mundos.

As respostas a esses desafios não começam do zero. O trabalho de instituições como a $\mathrm{OMS}^{6,8}$, de organismos de saúde pública ${ }^{7,12}$ que já passaram por experiências anteriores com pandemias e a literatura aportam contribuições que precisam ser valorizadas nesse momento ${ }^{11,13,14}$.

\section{Pandemia e Planos de resposta a pandemias}

Para a OMS, o Plano de resposta à COVID-19 e prontidão estratégica ${ }^{6}$ deve contemplar três objetivos: 1) Diminuir e interromper a transmissão, evitar epidemias e retardar sua transmissão; 2) Prover cuidado otimizado para todos os pacientes, em especial àqueles mais graves; e 3) Minimizar o impacto da epidemia sobre sistemas de saúde, serviços sociais e atividade econômica.

O documento descreve cenários de transmissão do vírus e propõe estratégias a serem adotadas para todos eles. A proteção da saúde dos trabalhadores da saúde e a segurança dos pacientes estão incluídas no 
terceiro objetivo, sendo que o seu alcance ótimo se mostra, umbilicalmente ligado, ao conteúdo de estratégias e atividades escolhidas para alcançar os dois primeiros e ao como são implementadas.

O PR à COVID-196 destaca:

COVID-19 é uma nova doença que é distinta de outras SARS, MERS e influenza. Embora o coronavírus e infecções pelo influenza possam se apresentar com sintomas similares, o vírus responsável pela COVID-19 é diferente com respeito à disseminação na comunidade e gravidade. Ainda há muito por ser descoberto sobre a doença e seus impactos em diferentes contextos. Preparação, prontidão, e ações de resposta continuarão a ser dirigidas pela rápida acumulação de conhecimento de saúde pública e científico. (p. 1, tradução nossa) ${ }^{6}$

A OMS ressalta o desconhecimento sobre a doença e diferenças identificadas em sua disseminação na comunidade e gravidade. É preciso considerar esses dois aspectos, monitorando a evolução da doença e o surgimento de novos conhecimentos com foco nos três objetivos do PR.

Nos EUA, o Centro de Controle de Doenças ${ }^{7}$ divulgou checklist de orientação sobre a preparação de hospitais para a pandemia. A composição do comitê de resposta destaca 15 atores (administração, controle de infecções, equipe médica, de enfermagem, cuidados intensivos, saúde ocupacional, entre outros). O checklist aborda estrutura para planejamento e tomada de decisões, desenvolvimento de plano escrito, elementos do plano incluindo: geral, comunicação, suprimentos e equipamentos médicos permanentes e de consumo, identificação e gestão de pacientes, acesso de visitantes e deslocamentos [na] instalação, saúde ocupacional, educação e treinamento, serviços de cuidados de saúde.

\section{Outras fontes úteis na montagem de resposta à pandemia}

Medidas de prevenção e controle de infecções são essenciais para evitar a disseminação da doença nos locais de trabalho. Destaque é dado a programa de treinamento da equipe ${ }^{15}$. O plano estratégico lista indicadores, cujo desempenho deve ser monitorado como parte de avaliação do plano de resposta e prontidão estratégica, ressaltando o percentual do total de casos que acometem trabalhadores da saúde ${ }^{8}$.

A importância atribuída à inclusão do tema saúde mental e aspectos psicossociais nos planos de resposta é crescente ${ }^{16-19}$.

A organização de planos de respostas à pandemia desperta interesse. A matriz de Haddon foi aplicada para diferentes tipos de ameaças, inclusive a pandemia do SARS, em 2003, destacando recomendações de preparo para fases pré, durante e pós-evento ${ }^{13}$. $\mathrm{O}$ modelo da gravata-borboleta também foi usado e mostra barreiras destinadas a evitar o evento, a perda de controle da situação e de eliminação e ou mitigação de suas consequências ${ }^{11}$. Artigo de revisão ${ }^{14}$ detalha medidas adotadas na organização da oferta do serviço, segundo fases da evolução da transmissão da doença, via casos importados e comunitária. O mesmo pode se falar de exemplos como o do Plano de resposta hospitalar ao COVID-1920, elaborado no âmbito do chamado Projeto Lean nas emergências.

O momento atual requer a síntese dessas diversas contribuições, com foco na proteção à saúde dos trabalhadores da saúde, considerando que a organização do funcionamento dos serviços e a antecipação de suas atividades nas diferentes fases da evolução da resposta à epidemia são fundamentais para o controle das infecções.

\section{Princípios éticos em tempos de crise}

Depois da pandemia H1N1, em 2009, foram sugeridos princípios de padrões de cuidados a serem adotados em contextos de crises. Tratam-se de esquemas de planejamento baseados em princípios éticos, regras legais, papel de fornecedores e engajamento da comunidade, pavimentando o caminho para decisões que permitam equidade e justiça na entrega de serviços médicos para aqueles que necessitam em situações de escassez de recursos ${ }^{9}$.

Os comitês de resposta à pandemia devem se guiar por critérios como justiça, dever de cuidar, dever de administrar recursos, de transparência, proporcionalidade e responsabilidade de modo a não desfavorecer os mais vulneráveis e a minimizar as chances de situações-limite ${ }^{9}$.

De modo similar são apontados três deveres éticos na provisão de cuidados de saúde em tempos de COVID-19: o dever de planejar, gerenciando incertezas; o dever de proteger, oferecendo suporte a trabalhadores e a populações vulneráveis; e o dever de orientar níveis de cuidados e padrões de cuidados em situações de crise ${ }^{10}$.

\section{Boas práticas e caminhos da prevenção}

As respostas dos serviços de saúde às mudanças requeridas pela evolução da pandemia, em especial no que se refere à abordagem dos três objetivos apontados no Plano de resposta à COVID-19 e prontidão estratégica ${ }^{6}$, estão intimamente associadas à 
prevenção. Diminuir a velocidade de transmissão e achatar a curva são medidas de eliminação de contatos em potencial pela redução da circulação de positivos assintomáticos e sintomáticos. O mesmo pode ser dito da coleta de dados por telefone, do uso de tele consultas e formulários eletrônicos. Iniciativas desse tipo também podem implicar mais tempo para a preparação do planejamento e das mudanças necessárias na infraestrutura (leitos de UTI, respiradores, EPI etc.) e em outros componentes do sistema (procedimentos, divisão do trabalho, aquisição de insumos etc.) a serem usados no enfrentamento da pandemia. Se essas medidas tiverem sucesso, pode ser minimizado o aumento do risco que tenderia a ocorrer com a sobrecarga da capacidade instalada no sistema em contexto de escassez de insumos e equipamentos necessários na resposta à doença.

A otimização dos cuidados ofertados a pacientes, em especial aos que se encontram em situação grave, também tende a se refletir na redução de impactos nocivos. Medidas de organização da assistência podem diminuir chances de contato entre pacientes suspeitos e ou confirmados de COVID-19 com pacientes internados e atendidos com outras patologias. O mesmo tende a acontecer com a seleção de salas e equipes exclusivas para pacientes da COVID-19.

O controle da circulação de visitantes e pacientes e a suspensão de procedimentos eletivos podem reduzir chances de infecção. De modo geral as equipes de segurança não conhecem a gama de atividades e rotinas existentes no serviço, cuja identificação revelaria novos alvos de prevenção. Para avançar nessa direção é preciso ouvir os trabalhadores.

Outros controles de engenharia incluem a provisão de condições para a higienização das mãos tanto na recepção como em outros pontos do serviço. Idem para espaços de espera, com ventilação abundante e natural e a realização de procedimentos geradores de aerossóis em isolamento respiratório, com pressão negativa e filtro high efficiency particulate arrestance (Hepa). Outras medidas, como ventilação-fluxo vertical laminar, aumento da taxa de ventilação e manutenção da umidade do ar elevada, podem ser associadas além do uso de rodo de UV-C para descontaminação de pisos, o robô de telepresença Double usado para fazer a triagem de pacientes, dentre outras inovações tecnológicas ${ }^{21-23}$.

A otimização de esquemas terapêuticos e da divisão de trabalho também podem diminuir tempos de internação e o número de expostos, reduzindo o risco de infecções. A realização de exames diagnósticos no próprio serviço pode reduzir impactos psíquicos associados a tempos de espera na incerteza. Em condições ideais, o serviço diminui as chances de entrar em colapso, minimizando riscos associados a sobrecarga de trabalho, desgaste, fadiga, precarização de condições de trabalho.

\section{Hierarquia de medidas de prevenção}

Vistas à luz dos critérios de hierarquia de medidas de prevenção, as providências citadas até o momento se referem a eliminação, controles de engenharia e medidas administrativas com foco na organização do trabalho. Medidas relativas a EPI devem se somar ao rol citado ${ }^{11}$.

Entre os problemas apontados em relação à gestão de EPI, é possível indicar que a maioria dos serviços não conseguiu se beneficiar de lições da pandemia de SARS, de 2003, se antecipando à escassez no mercado mundial. O mesmo se deu no tocante ao investimento em treinamentos adequados. Observação direta de práticas profissionais constatou que 90\% da equipe não seguiu a sequência ou técnica correta de colocação ou retirada de EPI ${ }^{11}$.

A desparamentação é crítica. É realizada depois que a tarefa de cuidado está concluída e facilita a baixa da guarda. Treinamentos devem alertar para isso e destacar possível contribuição da invisibilidade da ameaça, agravada pela possibilidade de transmissão por contato com superfícies inanimadas, inclusive do próprio EPI, que historicamente sempre aconteceu sem ser abordada como a ameaça atual.

Destacar apenas a disponibilização e a adequação do uso e retirada de equipamentos de proteção individual (EPI) como medidas de proteção à saúde dos trabalhadores da saúde, no mínimo, relativiza a importância a ser dada aos demais controles como de eliminação, de engenharia e administrativos na prevenção de infecções.

A Coordenação-Geral de Saúde do Trabalhador do Ministério da Saúde lançou Recomendações de proteção aos trabalhadores dos serviços de saúde no atendimento de COVID-19 e outras síndromes gripais ${ }^{21}$, em que destaca obrigações dos empregadores, medidas de controle de engenharia, de controle administrativo e orientações úteis em caso de infecção.

\section{O papel dos EPI}

O papel dos EPI na proteção à saúde dos trabalhadores da saúde na atual pandemia é ímpar. No caso do risco da contaminação biológica atuam como barreiras que podem evitar infecção.

Como os trabalhadores da saúde se infectam? Respostas apontam possíveis contribuições de 
falhas na colocação e retirada de EPI. O tempo, apontado como de 4 a até 6 dias em que portadores assintomáticos podem transmitir o vírus, também pode influenciar ${ }^{24}$.

Dificuldades na prevenção também foram destacadas e justificam a importância da defesa de comportamentos individuais de etiqueta e higiene de mãos.

Os cientistas encontraram substancial contaminação de equipamentos comuns em hospitais. Testes de swabs feitos em equipamentos de desinfecção de mãos e impressoras de autosserviço usadas por pacientes para imprimir cópias de seus relatórios médicos foram positivos em $20 \%$ dos testes. Teclados, maçanetas e mesas de trabalho também abrigavam o vírus, com $16 \%$ a 17\% dos swabs contendo traços do germe. Um em cada oito telefones também testaram positivo em swabs ${ }^{25}$.

Longe de poder indicar que a transmissão se deve, então, às falhas individuais de trabalhadores que não aderem ao suposto jeito certo e seguro de agir, os casos acima denunciam que os esforços anteriormente existentes nesses serviços em defesa da adoção de medidas de controle de infecções vinham sendo derrotados.

Atribuir a contaminação do trabalhador a erros que ele supostamente tenha cometido no uso de EPI é prática esperada em contextos em que prevalece a atribuição de culpa à vítima. E que assume pressuposto de que o uso sem erros sempre aconteceria se existisse prescrição que descrevesse a sequência de passos na colocação e retirada do equipamento.

Essa forma de pensar a aprendizagem é equivocada. Iniciante pouco experiente precisa focar a atenção no objetivo e nos gestos que precisa fazer e executará a tarefa de modo lento e sem a habilidade que só vai obter com a prática.

A lista de fatores que podem interferir na realização da tarefa e aumentar as chances de erros é enorme. O grupo e a história anterior do trabalhador influenciam o desempenho. O mesmo pode se dizer da situação. Se o trabalho está atrasado, se em momento de sonolência ou cansaço, se a sequência de gestos a ser usada no início da tarefa é a mesma de outra realizada com maior frequência, se há colegas aguardando o término do trabalho para que possam começar o deles, se durante a execução do trabalho acontece algo que é novo e não há ninguém próximo a quem se possa pedir ajuda, se há outro paciente precisando de cuidados, se há colega pedindo ajuda, tudo isso pode afetar o desempenho.

Estudos sobre erros humanos permitem hipótese de que o aumento dos apelos em defesa da adesão a práticas prescritas no contexto de pandemia é dirigido a coletivos de trabalhadores que historicamente, em sua maioria, resistiam à adesão ao que pareciam considerar exageros de cuidados a serem adotados. Aderir às medidas equivaleria em grande parte a nadar contra a corrente. Se a medida defendida é mais do mesmo que historicamente era rejeitado, se a percepção de perigo por parte daquele que decidiu reforçá-la é diferente daquela do trabalhador que deve aderir, há problema a ser enfrentado. E seu enfrentamento requer participação dos trabalhadores no processo de tomada de decisões. A medida inicial deve buscar pactuação, vista como compromisso que enfrente essa diferença de percepções e as razões históricas associadas à tolerância com os velhos padrões de comportamento ${ }^{26-29}$.

O planejamento dessa abordagem, além de requerer novos treinamentos que contemplem práticas observadas de colocação e retirada dos equipamentos, precisa abordar aspectos da natureza da nova doença que agravam os riscos de infecção em relação ao que se conhecia. Uma proposta interessante, com foco na proteção em ambiente hospitalar, foi recentemente disponibilizada em livre acesso pelo National Institute of Environmental Health Sciences ${ }^{30}$.

\section{A velocidade de origem de novos riscos é maior do que a da prevenção}

Treinamentos precisam chamar atenção para o fato de que a evolução da pandemia é mais rápida do que a das medidas de prevenção aos novos riscos. Participantes devem ser estimulados a estar atentos às novas situações de trabalho que precisam enfrentar ao colocar em execução a resposta do seu serviço à pandemia. Uma das tarefas de formação poderá ser a de recensear situações de risco criadas por interações surgidas no contexto da pandemia e identificar situações em que alguma coisa mudou no sistema e em que cabe a ele resolver a questão:

- Sente dificuldades que não existiam antes;

- Precisa fazer sozinho tarefa que antes era realizada com a ajuda de colegas;

- Precisa fazer o trabalho sem os equipamentos indicados como ideais;

- Precisa usar equipamentos e recursos novos e ou diferentes dos habituais;

- É forçado a fazer algo que deveria ser adiado, feito por outro colega ou com ajuda de colegas ou com materiais que não estão disponíveis;

- Precisa prolongar a jornada ou acelerar a forma de trabalho de modo não usual;

- Sem receber ordens de ninguém se sente forçado a fazer algo tendo que fazê-lo mais rápido e nem sempre com os recursos indicados. 
O objetivo buscado é desenvolver competências de reconhecimento de riscos ao lidar com situações de mudanças, identificando aquelas em que o risco de descontrole se torna maior, por exemplo, porque duas ou mais mudanças estão se encavalando ou porque precisa adotar posturas e gestos em situações de instabilidade e/ou em que se torna difícil manter o controle (ver, ouvir, sentir o cheiro) sobre o desenvolvimento da atividade. Ou ainda o jeito que estava habituado a fazer para resolver problemas não pode ser usado ou só pode com dificuldades antes não presentes. Enfim, situações que podem implicar aumento de riscos a serem manejados.

Em contexto de escassez têm sido defendidas estratégias de uso estendido e de reuso limitado de equipamentos. Isso é feito alertando para o aumento de riscos de contaminação e abrindo polêmicas. Tomadores de decisão precisam explicitar critérios usados nessas situações ${ }^{11}$.

Abordar esse tipo de problema no âmbito de plano de resposta de sistema à atual pandemia de COVID-19 é também reconhecer a falência de planejamento que permitisse ao serviço atuar em condições ótimas. Debater origens desse fracasso pode contribuir para a aprendizagem organizacional e minimização de problemas futuros. Explicitar custos humanos indesejados que de outra maneira seriam assumidos como decisões técnicas. Vale lembrar, decisões essas adotadas sem que que os principais afetados fossem ouvidos.

Referindo-se à escassez de pessoal e recursos em serviços que estão enfrentando a pandemia, a Dra. Susan Michie, da University College London, afirmou:

Nós estamos começando em posição perigosa. Nós não estamos em situação melhor por causas das medidas de austeridade. Para se ter tudo realmente limpo você precisa ter gente prestando atenção, você precisa ter bastante pessoal. É nesse quadro que a contaminação vai se dar ${ }^{25}$.

A possibilidade de interferências da precarização do trabalho foi destacada: "Hospitais do Reino Unido podem encarar problemas adicionais porque muitos deles contratam terceiros para limpeza, trabalhadores que, se adoecem, não recebem pagamento para os três primeiros dias" 25 .

Nessas condições, trabalhadores que começam a apresentar sintomas não se afastariam do trabalho podendo transmitir a doença.

No Brasil, parece importante explicitar como a crescente precarização do trabalho influencia comportamentos e afeta as possibilidades de transmissão da doença. O Sistema Único de Saúde (SUS) padece de subfinanciamento crônico agravado depois da Emenda Constitucional 95/2016 $6^{31}$, do teto de gastos, que alavancou brutal desfinanciamento do Sistema ${ }^{32}$. A advertência feita pela Dra. Michie vale para nós que também estamos começando em desvantagem.

$\mathrm{O}$ aumento de demandas a ser respondido em pandemias exige mudanças na orientação política neoliberal que continua resistindo aos esforços em defesa da vida. Socorro emergencial a bancos, ao sistema financeiro não suscita questionamentos do tipo "de onde virá o dinheiro?" ou "o que acontecerá depois?”.

Em entrevista ${ }^{33}$ ainda antes de ser empossado, o ex-ministro da saúde Nelson Teich, questionou investimento a ser feito na compra de respiradores necessários ao tratamento de doentes da COVID-19. E o fez em nome de suposta racionalidade econômica. Uma vez que, superada a crise, parte dos equipamentos poderia ficar ociosa.

Este texto destaca a importância da dimensão ética assumida em decisões de governantes e comitês de resposta à pandemia. Explicitar os interesses em disputa nas diferentes decisões é cuidado que precisa estar na agenda de todos. A natureza desse tipo de decisão não é de exclusiva racionalidade técnica.

Tempos de pandemia embutem oportunidades de aprendizagem e questionamentos sobre as lógicas assumidas nas escolhas adotadas. A crítica do ministro aceita como dado que a lógica da racionalidade econômica deva continuar prevalecendo no mundo pós-pandemia. Para dizer o mínimo, desconsidera vozes de pesquisadores dos mais diferentes campos do conhecimento, que alertam para a necessidade de cuidados especiais na abordagem dos impactos que afetam os mais vulneráveis, e de compreender a atual situação como ponto de corte na história mundial. Nada será como antes, é o que muitos anunciam. Em situações de crises complexas, como a da pandemia do SARSCoV-2, o uso exclusivo de orientações baseadas em evidências pode não ser suficiente ${ }^{34-36}$.

\section{Programa de respostas à pandemia $e$ a proteção à saúde dos trabalhadores da saúde}

Este texto aponta limites potenciais de programas de resposta à pandemia no tocante à proteção dos profissionais diretamente envolvidos no cuidado de saúde e em atividades de suporte, esboçando sugestões de como lidar com eles. Os princípios e ideias gerais apresentados também podem ser usados na prevenção em outras atividades de risco. A lista de potencialmente expostos inclui, entre outros, maqueiros, serviços de manutenção e limpeza, cuidadores de idosos, transporte, segurança pública, 
agentes funerários, sepultadores, professores, entregadores, jornalistas e todos aqueles em atividades definidas como essenciais e mantidas em operação.

Um dos limites possíveis é a ausência de abordagem de temas apontados como importantes para o plano, como, por exemplo, o das situações de saturação da capacidade instalada do serviço de saúde ou o da escassez de equipamentos de proteção. Visando minimizar esse problema, são sugeridos esforços de antecipação da identificação de piores cenários mostrados nas recomendações internacionais e na literatura.

Destaque é dado à natureza cambiante da pandemia e da velocidade com que evolui. Mudanças nas fases de transmissão da doença exigem adequações no plano de respostas. Todas as medidas adotadas pelo comitê de resposta à pandemia, todas as adequações assumidas, representam mudanças em relação ao modo como historicamente se dava o funcionamento do sistema. Essa situação em movimento ininterrupto se apresenta como desafio permanente.

A chegada da pandemia impõe mudanças nas atividades desenvolvidas no Sistema, que passa a atender número crescente de pessoas com doença nova e pouco conhecida. Todos os componentes do sistema reagem à nova situação. Surge o comitê de resposta, muda a estrutura de serviço (distribuição de leitos, fluxos etc.), surgem novos procedimentos, novas estratégias de aprendizagem, novas práticas assistenciais e de suporte. A divisão de trabalho passa por ajustes, as bases legais do funcionamento do serviço são modificadas, surgem interações com novos membros da comunidade.

As respostas à nova situação podem não ser adequadas, em parte, porque o tempo existente para o seu preparo e implementação foi muito curto e também por dificuldades e carências que o serviço já enfrentava.

É preciso freio de arrumação que estimule reflexões sobre a maneira como diferentes sistemas estão enfrentando a crise, alertando para a existência de recursos que podem ajudar os encarregados na construção dessas respostas e que alguns deles podem não ter sido identificados e incorporados no seu repertório de instrumentos.

O novo não prevalece sem resistências. A ameaça de intrusão de velhas práticas está presente. A evolução da pandemia e das respostas do sistema introduzem novas interações entre trabalhadores e entre estes e os materiais utilizados e ou com o seu contexto. Novas situações de exposição surgem tanto em situação de equilíbrio como de desequilíbrio entre demandas e capacidade instalada no sistema.

A pergunta a ser feita antes de cada decisão é "Estão sendo consideradas as implicações potenciais em termos de segurança do trabalho, segurança do paciente etc. ou há foco exclusivo naquilo que para o tomador era o problema a ser resolvido?".

Embora o não envolvimento de áreas diretamente afetadas pela decisão adotada seja visto como de potencial aumento de risco para os trabalhadores afetados o contrário também pode acontecer. Todas as decisões adotadas no sistema visando alcançar os dois primeiros objetivos do Plano de resposta à COVID-19 e prontidão estratégica ${ }^{4}$ podem contribuir para a proteção da saúde dos trabalhadores da saúde. Mesmo que não tenham sido tomadas pensando na prevenção.

A resposta mais frequente à pergunta acima diz que as repercussões em outras atividades e subsistemas não foram consideradas. A adoção de processos multivocais e participativos de tomada de decisões tende a exigir dos envolvidos esforços de antecipação de possíveis consequências e interferências em suas áreas de trabalho com as quais não estavam habituados a lidar. Erros podem e, provavelmente, devem acontecer. O esperado é que a prática ajude a aprimorar o uso desse modelo de decisões.

\section{Desafios do comitê de resposta à pandemia}

Um dos desafios do comitê de resposta à pandemia é o de acompanhar essas mudanças, identificando precocemente problemas não antecipados no plano e agilizando respostas cabíveis. Como fazer isso? A sugestão principal apresentada é a da montagem de linhas do tempo do desenvolvimento da pandemia e da resposta do sistema. Além de versões eletrônicas dessas linhas do tempo, a sala de controle do comitê deve ter versão impressa das mesmas constantemente atualizadas e discutidas.

Quais as principais fontes de mudanças a serem registradas nessas linhas? A resposta mais fácil se refere ao mapa das respostas do próprio comitê. Todas as decisões devem ser registradas e cotejadas com a evolução da epidemia. Modelos disponíveis de planos detalham o que priorizar, os elementos a serem contemplados. Cabe à equipe selecionar o que monitorar.

Da mesma maneira, checar com todas as áreas se houve mudança importante a registrar. Decisões importantes emanadas de componentes do sistema ou de organismos gestores municipais, estadual e ou federal também devem ser registradas e avaliadas.

No que se refere às mudanças na evolução da epidemia, recomenda-se a mesma lógica citada para os objetivos do plano. Todas as áreas devem ser consultadas sobre mudanças acontecidas em relação ao estágio anterior. Indicadores de 
acompanhamento da situação da pandemia e das respostas em desenvolvimento devem ser registrados e discutidos. Destaque para o percentual de casos afetando trabalhadores da saúde e também a acidentes e incidentes do trabalho afins à situação. Por quais razões? Em função do fato de que sua análise pode evidenciar dificuldades enfrentadas na execução do programa de respostas. Acidentes embutem avisos de problemas existentes no funcionamento do sistema.

Assim, o terceiro objetivo do Plano de resposta à COVID- $19^{6}$, lido em moldes que destacam a saúde do trabalhador da saúde, pode ser incluído na agenda do comitê de resposta à pandemia do sistema em questão.

\section{Considerações finais}

Este texto destaca a natureza cambiante da evolução da pandemia da COVID-19, natureza essa que precisa se manifestar nos planos de resposta à situação.

Identificar em tempo real o curso da evolução da doença nas diferentes realidades é um dos maiores desafios dos comitês de resposta à pandemia. Desafio que deve guiar a tomada de decisões relativas ao que fazer nos diferentes momentos.

Para a OMS, a resposta a esse tipo de questão deve estar ancorada em PR à doença, considerando três conjuntos de objetivos. Em situação em que podem se apresentar em aparente ou efetiva competição.

Nada é tão ruim que não possa piorar! E no momento atual, no Brasil, é crescente o número de notícias sobre serviços de saúde funcionando em sua capacidade máxima ou próximo disso. A ameaça de colapso da assistência bate à porta. A doença estaria próxima a passar da fase de transmissão comunitária para a de crescimento exponencial do número de

\section{Referências}

1. Koh D. Occupational risks for COVID-19 infection. Occup Med [Internet]. 2020 [citado em 5 maio 2020];70(1):3-5. Disponível em: https://academic. oup.com/occmed/article/70/1/3/5763894

2. Rothan HA, Byrareddy SN. The epidemiology and pathogenesis of coronavirus disease (COVID-19) outbreak. J Autoimmun [Internet]. 2020 [citado em 5 maio 2020];109:102433. Disponível em: https:// www.ncbi.nlm.nih.gov/pmc/articles/PMC7127067/

3. Organização Pan-Americana da Saúde. Folha informativa - COVID-19 (doença causada pelo novo coronavírus) [Internet]. Washington; 2020 casos e de óbitos. Do ponto de vista da saúde dos trabalhadores da saúde, o pior dos cenários!

Como essa situação pode se manifestar?

Um dos cenários é o de instalação simultânea de problemas. Se em contexto de efetivo reduzido somam-se aumento do número de afastamentos do trabalho por razões de saúde, aumento do número de doentes e do recurso a jornadas prolongadas, podem se somar cansaço, aumento na frequência de urgências e incidentes a serem enfrentados com menos chances da colaboração de colegas que também estejam sobrecarregados.

O sistema pode evoluir com degradação progressiva e pode piorar se o trabalho passa a ser feito em situação de escassez (vagas de UTI, leitos hospitalares, insumos, EPI etc.).

Tudo isso tende a aumentar as chances de contaminação e de vivências de sofrimento, de perdas, de frustrações, de impedimentos ao uso das melhores capacidades de equipes e serviços. $\mathrm{Na}$ pandemia atual relatos indicam que, no afã de proteger os seus entes queridos, trabalhadores estão se afastando de familiares, pessoas próximas e da rede de suportes com que contavam no cotidiano. $\mathrm{O}$ sofrimento mental encontra terreno fértil para prosperar. O trabalho atua como vetor na transmissão do vírus na sociedade!

Nesse cenário, em que se prenuncia o agravamento exponencial do quadro epidêmico, os planos de resposta vão passar pelo teste da vida.

Não há respostas mágicas para esse tipo de questões. Nesse texto, procurou-se destacar a necessidade de respostas que sempre considerem os três objetivos a buscar no desenvolvimento dos planos de resposta à epidemia. Caso contrário, as ameaças de custo humano crescente a ser pago pelas equipes de trabalhadores da saúde serão inevitáveis. [citado em 26 maio 2020]. Disponível em: https:// www.paho.org/bra/index.php?option $=$ com_content \&view $=$ article\&id =6101: covid19\&Itemid $=\overline{8} 75$

4. Brasil. Ministério da Saúde. Coronavírus Brasil. Painel de casos de doença pelo coronavírus 2019 (COVID-19) no Brasil pelo Ministério da Saúde [Internet]. Brasília; 2020 [citado em 26 maio 2020]. Disponível em: https://covid.saude.gov.br/

5. Programa Integrado em Saúde Ambiental e do Trabalhador. Boletim epidemiológico: doenças infecciosas e parasitárias relacionadas ao trabalho, incluindo a COVID-19. Centro Colaborador da 
Vigilância dos Agravos à Saúde do Trabalhador [Internet]. 2020 [citado em 29 maio 2020]; X(15):1-6. Disponível em: http://www.ccvisat.ufba.br/

6. World Health Organization. Critical preparedness, readiness and response actions for COVID-19 [Internet]. Genebra; 2020 [citado em 16 abr 2020]. Disponível em: https://www.who.int/publicationsdetail/critical-preparedness-readiness-andresponse-actions-for-covid-19

7. U.S Department of Health and Human Service. Centers for Disease Control and prevention. Comprehensive Hospital Preparedness Checklist for Coronavirus Disease 2019 (COVID-19) [Internet]. Washington;2020 [citado em 16 abr 2020]. Disponível em: https://www.cdc. gov/coronavirus/2019-ncov/downloads/HCW Checklist_508.pdf

8. World Health Organization. 2019 novel coronavirus (2019-nCoV): strategic preparedness and response plan [Internet]. Genebra; 2020 [citado em 29 maio 2020]. Disponível em: https://www. who.int/docs/default-source/coronaviruse/srp04022020.pdf?ua $=1$

9. Wong J, Goh QY, Tan Z, Lie SA, Shin Yi, et al. Preparing for a Covid-19 pandemic: a review of operating room outbreak response measure in a large tertiary hospital in Singapore. Can J Anesth [Internet]. 2020 [citado em 16 abr 2020];67(6):73245. Disponível em: https://link.springer.com/ article/10.1007/s12630-020-01620-9

10. Hick JL, Hanfling D, Wynia MK, Pavia AT. Duty to plan: health care, crisis standards of care, and novel coronavirus SARS-CoV-2. NAM Perspect [Internet]. 2020 [citado em 16 abr 2020]; discussion. doi: https://doi.org/10.31478/202003b

11. Berlinger N, Wynia M, Powell T, Hester M, Milliken A, et al. Ethical framework for health care institutions responding to novel coronavirus SARSCoV-2 (COVID-19): guidelines for institutional ethics services responding to COVID-19: managing uncertainty, safeguarding communities, guiding practice. Garrison: The Hastings Center; 2020 [citado em 18 abr 2020]. Disponível em: https:// www.thehastingscenter.org/wp-content/uploads/ HastingsCenterCovidFramework2020.pdf

12. European Centre for Disease prevention and Control. Preparedness planning for respiratory viruses in EU Member States. Three case studies on MERS preparedness in the EU. Estocolmo; 2015.

13. Barnett DJ, Balicer RD, Blodgett D, Fews AL, Parker CL, Links JM. The application of the haddon matrix to public health readiness and response planning. Environ Health Perspect. 2005;113(5):561-6.

14. Manton M, Johnson M, Scanlon M, Miles R, Cowley C. Bow tie for Covid-19 (as per CCPS/ EI guidance) [Internet]. New York: Center for Chemical Process Safety; 2020 [citado em 16 abr 2020]. Disponível em: https://www.aiche.org/sites/
default/files/html/544481/files/downloads/Bow\%20

Tie\%20for\%20Covid-19\%20-\%20EI\%20and\%20

CCPS\%20-\%20March\%2031,\%202020.pdf

15. Huh S. How to train health personnel to protect themselves from SARS-CoV-2 (novel coronavirus) infection when caring for a patient or suspected case. J. Educ Eval Health Prof [Internet]. 2020 [citado em 17 abr 2020];17:10. Disponível em: https://www.jeehp.org/DOIx.php?number $=357$

16. World Health Organization. Mental health and psychosocial considerations during the COVID-19 outbreak [Internet]. Genebra; 2020 [citado em 16 abr 2020]. Disponível em: https://www.who.int/ docs/default-source/coronaviruse/mental-healthconsiderations.pdf

17. Adams JG, Walls RM. Supporting the health care workforce during the COVID-19 global epidemic. JAMA [Internet]. 2020 [citado em 16 abr 2020];323(15):1439-40. Disponível em: https:// jamanetwork.com/journals/jama/fullarticle/2763136

18. Noal DS, Damásio F, coordenadores. Saúde mental e atenção psicossocial na pandemia covid-19: recomendações para gestores. Rio de Janeiro: Fiocruz; 2020.

19. Inter-Agency Standing Committee. Como lidar com os aspectos psicossociais e de saúde mental referentes ao surto de COVID-19: versão 1.5. Genebra; 2020.

20. Brasil. Ministério da Saúde. Acesse o plano de resposta hospitalar ao Covid-19 elaborado pelo projeto Lean nas emergências [Internet]. Brasília; 2020 [citado em 16 abr 2020]. Disponível em: https://setorsaude.com.br/acesse-o-plano-deresposta-hospitalar-ao-covid-19-elaborado-peloprojeto-lean-nas-emergencias/

21. Brasil. Ministério da Saúde. Coordenação Geral de Saúde do Trabalhador. Recomendações de proteção aos trabalhadores dos serviços de saúde no atendimento de COVID-19 e outras síndromes gripais. Brasília; 2020.

22. Universidade de São Paulo. USP entrega a hospital rodos com radiação ultravioleta para descontaminação [Internet]. São Paulo: Jornal da USP; 2020 [citado em 16 maio 2020]. Disponível em: https://jornal.usp.br/ciencias/ciencias-exatase-da-terra/usp-entrega-a-hospital-rodos-comradiacao-ultra-violeta-para-descontaminacao/

23. Tito F. Robôs ajudam no combate e prevenção contra o coronavírus; fotos [Internet]. Rio de Janeiro: G1; 2020 [citado em 16 maio 2020]. Disponível em: https:/g1.globo.com/bemestar/ coronavirus/noticia/2020/03/17/robos-ajudamem-combate-e-prevencao-contra-o-coronavirusfotos.ghtml

24. Arons MM, Hatfield KM, Reddy SC, Kimball A, James A, et al. Presymptomatic SARS-CoV-2 infections and transmission in a skilled nursing facility. N Engl J Med [Internet]. 2020 [citado em 5 maio 2020];382:2081-90. Disponível em: https:// www.nejm.org/doi/full/10.1056/NEJMoa2008457 
25. The Guardian. Call to protect hospital staff from coronavirus contamination [Internet]. Londres; 2020 [citado em 17 abr 2020]. Disponível em: https://www.theguardian.com/society/2020/mar/17/ urgent-call-to-protect-hospital-workers-fromcoronavirus

26. Amalberti R. Gestão da segurança: teorias e práticas sobre as decisões e soluções de compromisso necessárias. Presidente Prudente: Gráfica CS; 2016.

27. Dekker S. Drift into failure: from hunting broken components to understanding complex systems. Farnham: Ashgate; 2011.

28. Dekker S. Patient safety: a human factors approach. Boca Raton: CRC Press; 2011

29. Woods D, Dekker S, Cook R, Johannensen L, Sarter N. Behind Human Error. Farnham: Ashgate; 2010.

30. National Institute of Environmental Health Sciences. Protecting Yourself from COVID-19 in the Workplace (IMAGE) [Internet]. Local de publicação; 2020 [citado em 26 maio 2020]. Disponível em: https://www.eurekalert.org/ multimedia/pub/227585.php

31. Brasil. Emenda Constitucional no 95, de 15 de dezembro de 2016: altera o Ato das Disposições Constitucionais Transitórias, para instituir o Novo Regime Fiscal, e dá outras providências [Internet]. Brasília; 2016 [citado em 29 maio 2020]. Disponível em: http://www.planalto.gov.br/ ccivil_03/constituicao/emendas/emc/emc95.htm
32. Mehry EE, Bertussi DC, Santos MLM. Merhy, Bertussi e Santos: não basta acesso universal; é preciso um sistema universal de saúde [Internet]. São Paulo: Vi o Mundo; 2020 [citado em $18 \mathrm{abr}$ 2020]. Disponível em: https://www.viomundo.com. br/blogdasaude/merhy-bertussi-e-santos-nao-bastaacesso-universal-e-preciso-um-sistema-universalde-saude.html

33. Instituto Oncoguia. $9^{\circ}$ Fórum Nacional Oncoguia: complexidade do sistema de saúde brasileiro: Instituto Oncoguia [Internet]. São Paulo; 2020 [citado em 10 maio 2020]. Disponível em: http:// www.oncoguia.org.br/oncoguia-tv/9-forumnacional-oncoguia-complexidade-do-sistema-desaude-brasileiro-instituto-oncoguia/655/70/

34. Agamben G, Zizek S, Nancy JL, Berardi FB, Petit SL, Butler J, et al. Sopa de Wuhan: ASPO (Aislamiento Social Preventivo y Obligatorio) [Internet]. São Paulo: Canal Contemporâneo; 2020 [citado em 2 jun 2020]. Disponível em: http://www.canalcontemporaneo.art.br/quebra/ archives/009872.html

35. Mike D, Žižek S, Badiou A, Davis M, Bihr A, Zibechi R. Coronavírus e a luta de classes. Brasília: Terra Sem Amos; 2020.

36. Poole DN, Escudero DJ, Gostin LO, Leblang D, Talbot EA. Responding to the COVID-19 pandemic in complex humanitarian crises. Int J Equity Health [Internet]. 2020 [citado em 2020 abr 18];19:41. Disponível em: https://equityhealthj.biomedcentral. com/articles/10.1186/s12939-020-01162-y 